\title{
The current status of the Nano-JASMINE project
}

\section{Y. Kobayashi ${ }^{145}$, N. Gouda ${ }^{15}$, T. Yano $^{1}$, M. Suganuma ${ }^{1}$,} M. Yamauchi ${ }^{14}$, Y. Yamada ${ }^{3}$, N. Sako ${ }^{2}$ and S. Nakasuka ${ }^{2}$

${ }^{1}$ National Astronomical Observatory of Japan, 2-21-1 Osawa, Mitaka, Tokyo, 181-8588 Japan

${ }^{2}$ School of Engineering, The University of Tokyo, 7-3-1 Bunkyoku, Hongo, Tokyo, Japan

${ }^{3}$ Faculty of Science, Kyoto University Kitashirakawa, Oiwakecho, Sakyoku, Kyoto, Japan

${ }^{4}$ Department of Astronomy, The University of Tokyo, 7-3-1 Bunkyoku, Hongo, Tokyo, Japan

${ }^{5}$ The Graduate University for Advanced Studies 2-21-1, Osawa, Mitaka, Tokyo, Japan

\begin{abstract}
Nano-JASMINE is a nano-size astrometry satellite that will carry out astrometry measurements of nearby bright stars for more than one year. This will enable us to detect annual parallaxes of stars within 300 pc from the Sun. We expect the satellite to be launched as a piggyback system as early as in 2009 into a Sun synchronized orbit at the altitude between 500 and $800 \mathrm{~km}$. Being equipped with a beam combiner, the satellite has a capability to observe two different fields simultaneously and will be able to carry out HIPPARCOS-type observations along great circles. A $5 \mathrm{~cm}$ all aluminum made reflecting telescope with a aluminum beam combiner is developed. Using the on-board CCD controller, experiments with a real star have been executed. A communication band width is insufficient to transfer all imaging data, hence, we developed an onboard data processing system that extracts stellar image data from vast amount of imaging data. A newly developed $2 \mathrm{~K} \times 1 \mathrm{~K}$ fully-depleted $\mathrm{CCD}$ will be used for the mission. It will work in the time delayed integration(TDI) mode. The bus system has been designed with special consideration of the following two points. Those are the thermal stabilization of the telescope and the accuracy of the altitude control. The former is essential to achieve high astrometric accuracies, on the order of 1 mas. Therefore relative angle of the beam combiner must be stable within 1 mas. A 3-axes control of the satellite will be realized by using fiber gyro and triaxial reaction wheel system and careful treatment of various disturbing forces.
\end{abstract}

Keywords. space vehicles, instrumentation: detectors

\section{Introduction}

It is possible to realize a low cots satellite mission within a short development period, if the satellite is physically small and if a piggy-back launching is employed. A twodimensional array detector enables us to perform efficient observations, and a larger number of stars can be observed than that observed by Hipparcos. By combining the data in the Hipparcos catalog, we can determine proper motions with accuracies as high

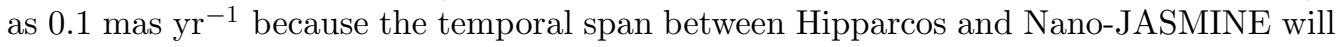
exceed 15 years.

\section{Current status}

We have performed evaluation experiments for a prototype system. By using the NanoJASMINE simulator, we have evaluated altitude control strategies in both the initial and normal operation phases. The results of thermal analysis for the Nano-JASMINE structure will be used to improve the final design of the satellite. 
The optics for the 5-cm telescope are manufactured by using super-precision diamondturning machines. These optics form a Ritchey-Chrétien-type optical system that has a composite F-ratio of 33, which matches the pixel size of a CCD. We have achieved sufficient optical performance for this telescope(Suganuma et al. 2008). A fully-depleted CCD that has been developed by Hamamatsu Photonics K.K. will be used for NanoJASMINE. We have been developing a sufficiently small controller that can control the CCD in the TDI operation mode. Since the data transfer rate is limited, we have been developing a hardware stellar image extractor that can work sufficiently fast to be operated in real time. The system is constructed on a FPGA system, and a new algorithm has been developed for this system(Yamauchi et al. 2008). The mission control computer will be constructed on a FPGA system that has a CPU core. The prototype system has been subjected to integral experiments.

The entire satellite system is controlled in the concentrated mode by a FPGA controller that has a CPU core. Li-ion batteries are used for the power unit and a mutual surveillance system maintains its stability. A large area is reserved for solar batteries so that the required power can be maintained; this increases the size of the satellite. The satellite has magnetic sensors, fiber optical gyros, a star tracker for altitude sensing, and a magnetic torquer and reaction wheel system for the altitude actuator. A data transmitter will support more than $3 \mathrm{kbps}$ in the worst case.

Since the structural deformation of optics due to the changes in temperature will degrade the accuracy of astrometric measurements, we have performed thermal analysis. Reducing the temperature gradient in the telescope structure is most important. We have taken care of the telescope's thermal insulation and have increased its capacity to endure a large amount of heat conduction. Our analysis shows that the deformation of the beam combiner, which is the most sensitive component with respect to measurement accuracy, is less than 0.7 mas. The thermal radiation efficiency is also important to maintain the CCD at low temperatures.

The altitude control of Nano-JASMINE is one of the major challenges. We have studied the methods to form an operational sequence by using the Nano-JASMINE simulator. In the first stage of operations, when the satellite altitude is significantly displaced from the expected position, we will operate the Magnetic Torquer with reference to the outputs of magnetic sensor. In the next stage, we will use the Fiber Optical Gyro and Star Tracker as altitude sensors and a 3 -axes reaction wheel to control the altitude. In the final stage, we will use the CCD outputs. Elongated stellar images will indicate on the altitude error.

We have not yet finalized the launcher. Among others HIIa and Cyclone4 are being considered for the launcher. The satellite will be launched into a sun-synchronized orbit at the altitude range between $500 \mathrm{~km}$ and $800 \mathrm{~km}$. The other orbital parameters depend on the major satellite requirements. We will optimize the survey parameters for different orbital parameters. The date of the launch is not yet fixed. It can be as early as 2009 .

\section{References}

Suganuma, M. et al. 2008, Proc. IAU symposium No. 248, this volume p. 284

Yamauchi, M. et al. 2008, Proc. IAU symposium No. 248, this volume p. 294 